\title{
Calmodulin-Like Skin Protein (CLSP) is a Novel Biomarker Candidate for Pick's Disease by Unfoldin-Modified Proteomic Analysis
}

Kana Nishijima, Kaoru O'hara, Kiyotoshi Kaneko and Naomi Hachiya*

Department of Neurophysiology, Tokyo Medical University, Tokyo 160-8402, Japan

\begin{abstract}
We identified a biomarker candidate for Pick's disease using robust protein-unfolding activity, designated Unfoldin, which was purified from yeast cells. Unfoldin localized to the cell periphery at the stationary phase, whereas it was on the budding sites during the log phase and then surrounded the bud neck during cell division. ATP, but not its hydrolysis, promoted Unfoldin binding to protein substrates and unfolded their conformation. A total of 514 Pick bodies were purified and isolated with a laser-microdissection system without a healthy brain region and solubilized with Unfoldin in the presence of ATP, and then the components were analyzed using a shotgun protein analysis by liquid chromatography-tandem mass spectroscopy. A MASCOT analysis revealed that calmodulin-like skin protein is a possible marker protein for Pick's disease, and it was localized in the Pick bodies of a patient with Pick's disease. Unfoldin will open the way to diagnostics and therapeutics, particularly for protein-aggregation related diseases.
\end{abstract}

Keywords: Pick's disease; Biomarker; CLSP; Aggregation; Neurodegenerative disease

\section{Introduction}

Pick's disease, a type of progressive presenile dementia that affects brain function, eventually causes loss of verbal skills and problem-solving ability [1]. It accounts for $5 \%$ of all dementias and is neuropathologically characterized by distinct tau-immunoreactive intraneuronal inclusion bodies known as Pick bodies (PBs) [2]. Abnormally phosphorylated tau proteins have been detected from total brain homogenates [2-4], and we previously detected abnormally phosphorylated tau proteins from only one $\mathrm{PB}$, which was isolated using a laser-microdissection system and oligomeric Aip2p/Unfoldindependent solubilization [5,6]; however, no further biomarker has been identified to date.

To identify a novel biomarker for Pick's disease, we used the robust protein-unfolding activity from Saccharomyces cerevisiae, designated as Unfoldin. Unfoldin solubilized the isolated and purified PBs, which were insoluble with any detergent or solubilizing agent, and the following experiments, using liquid chromatography-tandem mass spectroscopy (LC-MS/MS) revealed that the calmodulin-like skin protein (CLSP) [7] is a novel biomarker candidate of Pick's disease.

\section{Methods}

\section{Yeast strains and antibody}

Protease-deficient strain SH2777 for Unfoldin purification was a gift from Dr. Harashima, Osaka University. To prepare the antibody for the yeast alpha-factor, E. coli expressed and purified protein was used as the antigen, and the antibody was affinity purified using this antigen. A synthetic peptide, corresponding to the 15 amino acid residues at the C-terminal of actin-interacting protein2 (Aip2p) (VHYDPNGILNPYKYI), was coupled through a C-terminal cysteine residue to bovine serum albumin. Anti-tau8 (phosphorylationdependent monoclonal antibody specific to phosphorylated Ser202/ Thr205) was purchased from Thermo Scientific (Rockford, IL, USA). Rabbit polyclonal antibody against CLSP/CALML5 was purchased from Proteintech (Chicago, IL, USA).

Trypsin-susceptibility assay for purifying protein-unfolding activity

For the substrate protein-unfolding assay using trypsin susceptibility, the gene fragment of hexa hisitidine-tagged prepro alphafactor ( $\mathrm{ppaF}$ ) of Saccharomyces cerevisiae was amplified by polymerase chain reaction (PCR), inserted into the pET 11a plasmid, expressed in $E$. coli BL21(DE3) using a pET system, and purified. The purified ppaF was dialyzed against buffer A (10 mM Hepes-KOH, pH 7.4, 1 $\mathrm{mM}$ DTT, and $\left.1 \mathrm{mM} \mathrm{Mg}(\mathrm{OAc})_{2}\right)$. Yeast microsomes were prepared using protease-deficient strain SH2777. Microsomes were treated with buffer A containing $0.5 \mathrm{M} \mathrm{NaCl}$ on ice for $20 \mathrm{~min}$ and then centrifuged at $100,000 \times \mathrm{g}$ at $4^{\circ} \mathrm{C}$ for $60 \mathrm{~min}$ to prepare the salt extract fraction. After centrifugation, the salt extract fraction was dialyzed against buffer A overnight at $4^{\circ} \mathrm{C}$. The assay $(200 \mu \mathrm{l})$ was started by adding $0.1 \mu \mathrm{g}$ of ppaF to buffer A, containing $1 \mathrm{mM}$ ATP and microsome salt extracts, and incubated at $30^{\circ} \mathrm{C}$ for $15 \mathrm{~min}$. Then, samples were treated with a low concentration of trypsin $\left(0.2 \mu \mathrm{g} \mathrm{ml}^{-1}\right)$ at $16^{\circ} \mathrm{C}$ for 15 $\mathrm{min}$. The reaction was stopped by adding soybean trypsin inhibitor 0.4 $\mu \mathrm{g} \mathrm{ml}^{-1}$ ) on ice for $5 \mathrm{~min}$ followed by addition of trichloroacetic acid (TCA) for the precipitation of samples with a tRNA carrier. Then the solution was subjected to sodium dodecyl sulfate-polyacrylamide gel electrophoresis (SDS-PAGE) and immunoblotting. An affinity purified polyclonal rabbit anti- pro $\alpha \mathrm{F}$ antibody was used as the first antibody, and a horseradish peroxidase-linked IgG (MP Biomedical, Solon, $\mathrm{OH}$, USA) was used as the secondary antibody to detect ppaF.

\section{Purification of hexa histidine-tagged Unfoldin}

We prepared the Unfoldin expression strain in yeast under the ADH promoter to obtain sufficient Unfoldin. The hexa-histidine tagged YDL178w gene was amplified by PCR and inserted into the pAUR123 aureobasidin $\mathrm{A}(\mathrm{AbA})$ selective expression vector (Takara Bio Inc.,

*Corresponding author: Naomi Hachiya, Department of Neurophysiology, Tokyo Medical University, Tokyo 160-8402, Japan, Tel: 81-3-3351-6141; Fax: 81-3-3351-6544; E-mail: naomi@tokyo-med.ac.jp

Received July 09, 2012; Accepted August 27, 2012; Published August 28, 2012

Citation: Nishijima K, O'hara K, Kaneko K, Hachiya N (2012) Calmodulin-Like Skin Protein (CLSP) is a Novel Biomarker Candidate for Pick's Disease by UnfoldinModified Proteomic Analysis. J Neurol Neurophysiol S11-003. doi:10.4172/21559562.S11-003

Copyright: (c) 2012 Nishijima K, et al. This is an open-access article distributed under the terms of the Creative Commons Attribution License, which permits unrestricted use, distribution, and reproduction in any medium, provided the original author and source are credited. 
Shiga, Japan). The SH2777 protease-deficient strain was transformed with this plasmid, and transformants were grown on a $0.5 \mu \mathrm{g} \mathrm{ml}^{-1} \mathrm{AbA}$ containing YPD plate and inoculated $(8 \mathrm{~L})$ at $30^{\circ} \mathrm{C}$ overnight to an optical density value of 0.8 . The cells were suspended in four volumes of buffer B (50 mM NaPi, pH 8.0, $150 \mathrm{mM} \mathrm{NaCl}, 10 \mathrm{mM}$ imidazole), crushed with glass beads, and centrifuged at $10,000 \mathrm{rpm}$ for $10 \mathrm{~min}$ at $4^{\circ} \mathrm{C}$. The supernatants were collected and ultra-centrifuged at 100,000 $\times \mathrm{g}$ and $4^{\circ} \mathrm{C}$ for $1 \mathrm{hr}$. The precipitate was resuspended and subjected to a Ni-NTA agarose column (Qiagen, Valencia, CA, USA), equilibrated in buffer $\mathrm{B}$, and eluted with buffer $\mathrm{B}$ containing $0.5 \mathrm{M}$ imidazole. The eluted active fractions were dialyzed against buffer $\mathrm{C}$ (10 mM Hepes$\mathrm{KOH}, \mathrm{pH} 7.4,50 \mathrm{mM} \mathrm{NaCl}, 1 \mathrm{mM}$ DTT), subjected to an ion-exchange Mono Q column (AKTA system; GE Healthcare, Parsippany, NJ, USA), equilibrated with buffer $C$, and eluted in a linear gradient of 100-500 $\mathrm{mM} \mathrm{NaCl}$. The biologically active eluted fractions were dialyzed against buffer D (50 mM NaPi, pH 7.5, $10 \mathrm{mM} \mathrm{NaCl}, 1 \mathrm{mM} \mathrm{Mg}(\mathrm{OAc})_{2}$ ), and subjected to gel filtration on a Superdex 200 column equilibrated with buffer D. Purity was evaluated by silver staining and Western blotting.

\section{Immunofluorescence microscopy and low-angle rotary shadowing electron microscopy}

Immunofluorescence microscopy was performed using standard protocols and methanol/acetone fixation. Since Unfoldin consisted of Aip2p, the affinity-purified Unfoldin/Aip2p peptide antibody was diluted 1:100. An Alexa 488-conjugated anti-rabbit IgG secondary antibody (Molecular Probes, Eugene, OR, USA) was diluted 1:200 for Unfoldin localization. Samples were imaged with the Delta-Vision microscopy system (Applied Precision, Issaquah, WA, USA), which included an Olympus IX70 inverted microscope with a 100x objective, and the out-of-focus light of the visualized images was removed by interactive deconvolution. Low-angle rotary shadowing microscopy was performed as described previously [8].

\section{Immunohistochemistry}

After informed consent was obtained, tissue samples (frontal cortex from a patient with Pick's disease; female, 71 years old) were placed in a deep freezer at Nippon Medical University. Cryostat sections $(5 \mu \mathrm{m}$ thick) were prepared from the frozen brain tissue, fixed in $4 \%$ paraformaldehyde at room temperature for $15 \mathrm{~min}$, and immunostained using the avidin-biotin-peroxidase complex (ABC) method with a Vectastatin ABC kit (Vector Laboratories, Burlingame, CA, USA). The mouse monoclonal antibody against phosphorylationdependent tau protein AT8 was used at a 1:200 dilution, and the rabbit polyclonal antibody against CLSP/CALML5 was used at a 1:500 dilution. Procedures were in accordance with the institutional ethical standards on human experimentation. For the double staining, tissue sections were incubated with primary antibodies and washed four times with distilled water and PBS then incubated with biotinylated antimouse and anti-rabbit secondary antibodies for $30 \mathrm{~min}$. All secondary antibodies were obtained from Jackson ImmunoResearch Laboratories (West Grove, PA). After being washed four times with PBS, specimens were incubated with avidin/biotinylated horseradish peroxidase (HRP). Development was done using the 3,3'-diaminobenzidine (DAB) Substrate Kit for Peroxidase (Vector Laboratories, Burlingame, CA) and observed by light microscopy OLYMPUS IX71 with CCD camera.

\section{Proteomic analysis of laser-micro dissected PBs following Unfoldin pretreatment}

A total of 514 laser micro-dissected PBs were incubated with Unfoldin, as described previously [5,6]. After Unfoldin pretreatment,
PBs were analyzed using liquid chromatography-tandem mass spectroscopy (LC-MS/MS) shotgun proteomics methods following a MASCOT analysis.

\section{Results}

We developed an in vitro protein-unfolding assay system using the factor-dependent increase in protease susceptibility of the substrate as a measure to identify robust protein-unfolding activity and serendipitously isolated ATP-dependent activity. We used the yeast pheromone pre-pro alpha factor ( $\mathrm{ppaF})$, which is transported posttranslationally across the endoplasmic reticulum $[9,10]$ membrane in a cytosolic factor-dependent manner, as the substrate. The salt extract of the yeast microsomal fraction increased trypsin-susceptibility, otherwise it was undigested in a dose-dependent manner (Figures 1 and 2), suggesting that the microsomal salt extract contained the activity that unfolds the conformation of the substrate protein to increase protease susceptibility. We purified a $58 \mathrm{kDa}$ protein (Figure 3) with this activity to homogeneity using several ion-exchange chromatography steps and adsorption matrices, followed by affinity chromatography with an ATP-agarose column (Table 1).

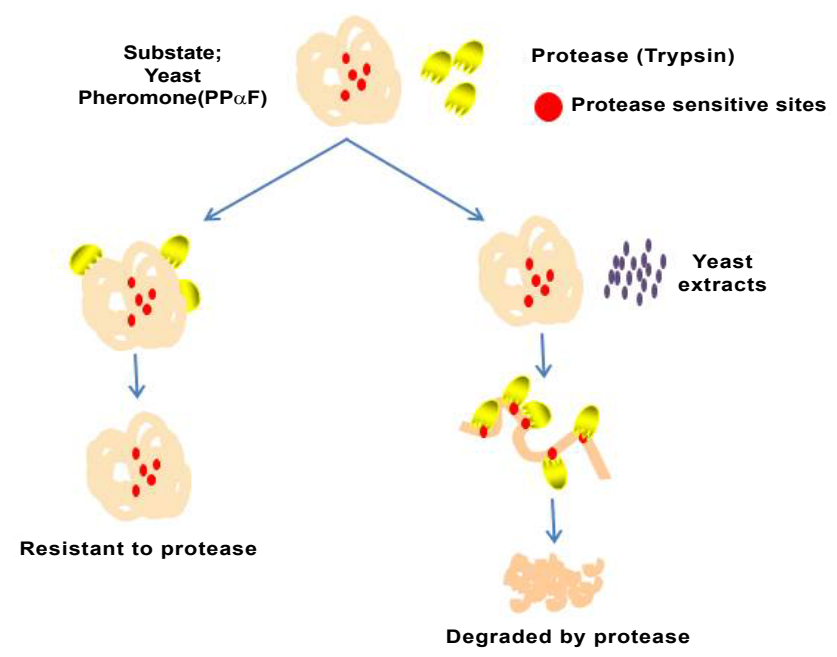

Figure 1: Trypsin susceptibility assay scheme. The salt extract of the yeas microsomal fractions increased trypsin susceptibility of prepro alpha-factor (ppaF).

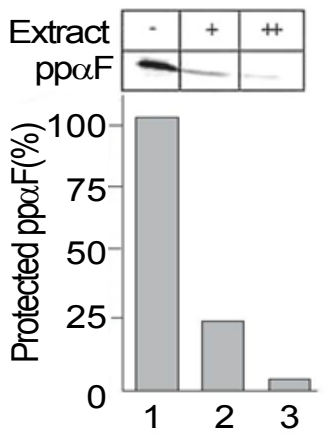

Figure 2: Yeast microsome salt extract fraction contains protein-unfolding activity. The salt extract (-) is shown as a trypsin protected control band and exhibited at $100 \%$. (+), $50 \mu \mathrm{g} ;(++), 100 \mu \mathrm{g}$ of crude extract. The protected bands were detected by immunoblotting using polyclonal antibody against $\alpha F$ and visualized by ECL-plus. 
The purified factor exhibited robust protein-unfolding activity in vitro. Amino acid sequence analysis revealed that the purified factor was identical to the product of the YDL178w yeast open reading frame identified as Aip2p/Dld2p [11,12]; however, it eluted as a single peak on gel-filtration chromatography with an apparent molecular weight of $670 \mathrm{kDa}$ [13-15]; hence, we hereafter designated the activity consisting of an oligomeric form as "Unfoldin". Because the molecular weight of the monomeric form (Aip2p/Dld2p) was $\sim 60 \mathrm{kDa}$, as estimated by SDS-PAGE (Figure 3 ) and amino acid sequence analysis, it was obvious that Unfoldin formed a homo-oligomer of 10-12 subunits. In good accordance with this result, low-angle shadowing electron microscopy of recombinant Unfoldin revealed that it was an oval-ring shaped oligomeric structure $\sim 10 \mathrm{~nm}$ in diameter with a $\sim 2 \mathrm{~nm}$ central cavity. ATP regulates opening and closing of the "gate" that forms the opening within Unfoldin (Figure 4) [14]. We prepared a specific antibody against a synthetic peptide corresponding to the C-terminal 15 amino acid residues of Aip2p/Dld2p to investigate intracellular localization of Unfoldin in vivo at different yeast cell growth stages using indirect immunofluorescence microscopy. Cell growth was synchronized by adding yeast alpha factor. As shown in Figure 5, Unfoldin localized in the plasma membrane periphery at the stationary phase, whereas it was concentrated to small buds or localized to an area surrounding the bud-neck during the log phase. These observations suggest that Unfoldin functions during actin movement, particularly [16] during bud emergence or cell division processes.

We previously reported that only one PB was sufficient to illustrate an immunoblot signal in the presence of Unfoldin, indicating that pretreatment with Unfoldin strongly enhances immunoblotting $[5,6]$. Therefore, to identify a novel biomarker protein from PBs, we isolated 514 PBs from frozen brain frontal lobe tissue of a patient with Pick's disease without a healthy region using a laser micro-dissection system and incubated it with $500 \mathrm{ng}$ of Unfoldin in the presence of ATP at $30^{\circ} \mathrm{C}$ for $90 \mathrm{~min}$. The solubilized sample was analyzed using a LC-MS/MS shotgun system. Among the identified proteins (Table 2), we focused on calmodulin-like skin protein (CLSP) [7], because it interacts with transglutaminase3 (TGase3) in the human brain, which is related to neurodegenerative diseases $[11,12]$ and examined frontal lobe brain tissue from a patient with Pick's disease by immunohistochemistry using nonfluorescent labeling technique [17].

Briefly, tissue sections were incubated with primary antibodies (The mouse monoclonal antibody against phosphorylationdependent tau protein AT8 was used at a 1:200 dilution, and the rabbit polyclonal antibody against CLSP was used at a 1:500 dilution) then washed and incubated with biotinylated anti-mouse and anti-rabbit secondary antibodies for $30 \mathrm{~min}$. After being washed four times with PBS, specimens were then incubated with avidin/biotinylated horseradish peroxidase (HRP). Development was done using the 3,3'-diaminobenzidine (DAB) Substrate Kit for Peroxidase (Vector Laboratories, Burlingame, CA) and observed by light microscopy OLYMPUS IX71 with CCD camera.

As shown in Figure 6, phosphorylated tau protein (brown color, panels A, D, G, and J) and CLSP (purple color, panels B, E, H, and K) were merged on PBs (panels C, F, I and L), suggesting that CLSP is possible biomarker for Pick's disease.

\section{Discussion}

Millions of people worldwide suffer from neurodegenerative disorders. Most of these illnesses manifest themselves later in life. The number of people affected with these diseases will grow, considering

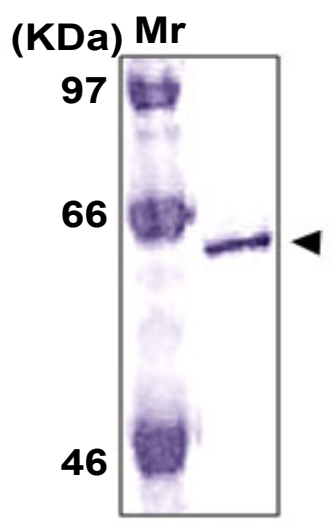

Figure 3: Sodium dodecyl sulfate-polyacrylamide gel electrophoresis (SDSPAGE) and Coomassie Brilliant Blue (CBB) staining of purified Unfoldin. Unfoldin $(150 \mu \mathrm{g})$ was subjected to SDS-PAGE and stained with CBB.
Closed state

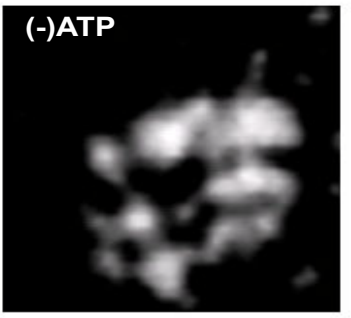

Open state

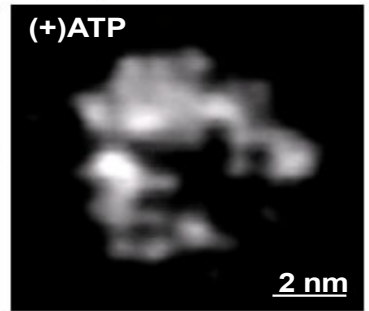

Figure 4: Unfoldin consisted of a homo-oligomer. Purified hexa histidinetagged Unfoldin $\left(100 \mu \mathrm{g} \mathrm{ml}^{-1}\right)$ was prepared and used as a specimen for lowangle shadowing electron microscopy.

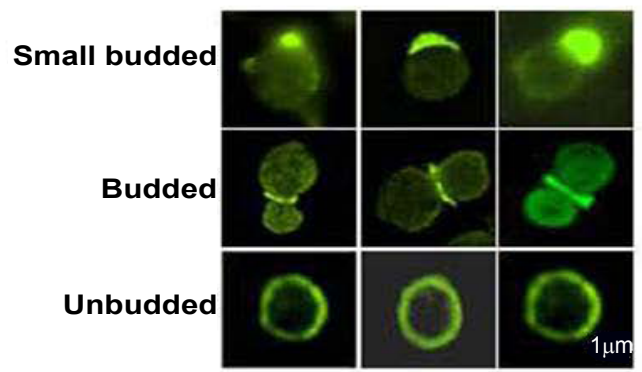

Figure 5: Unfoldin localization in vivo. Yeast cells were cultured to an optical density (OD) value at $600 \mathrm{~nm}$ of $0.05-0.1$ (middle panels), $0.5-0.8$ (upper panels), or $5-8$ (lower panels) at $30^{\circ} \mathrm{C}$ to immunostain endogenous Unfoldin. Immunofluorescence staining was performed using standard protocols, and the anti-Unfoldin specific antibody was used at a 1:100 dilution. FITC-conjugated goat anti rabbit IgG was used as the secondary antibody at a 1:200 dilution. Each sample was examined with Delta-Vision microscopy. Out-of-focus light was removed by interactive deconvolution.

the increase in life expectancy. However, because of limited sample availability and excess aggregation properties of disease-related inclusion bodies, most of the disease-causing proteins have not been identified, and their functions are mostly unknown. Furthermore, due to the highly aggregated property of proteins, neuronal inclusion bodies, particularly those isolated by laser micro-dissection, are often too disrupted for analysis. Even worse, conventional procedures, 

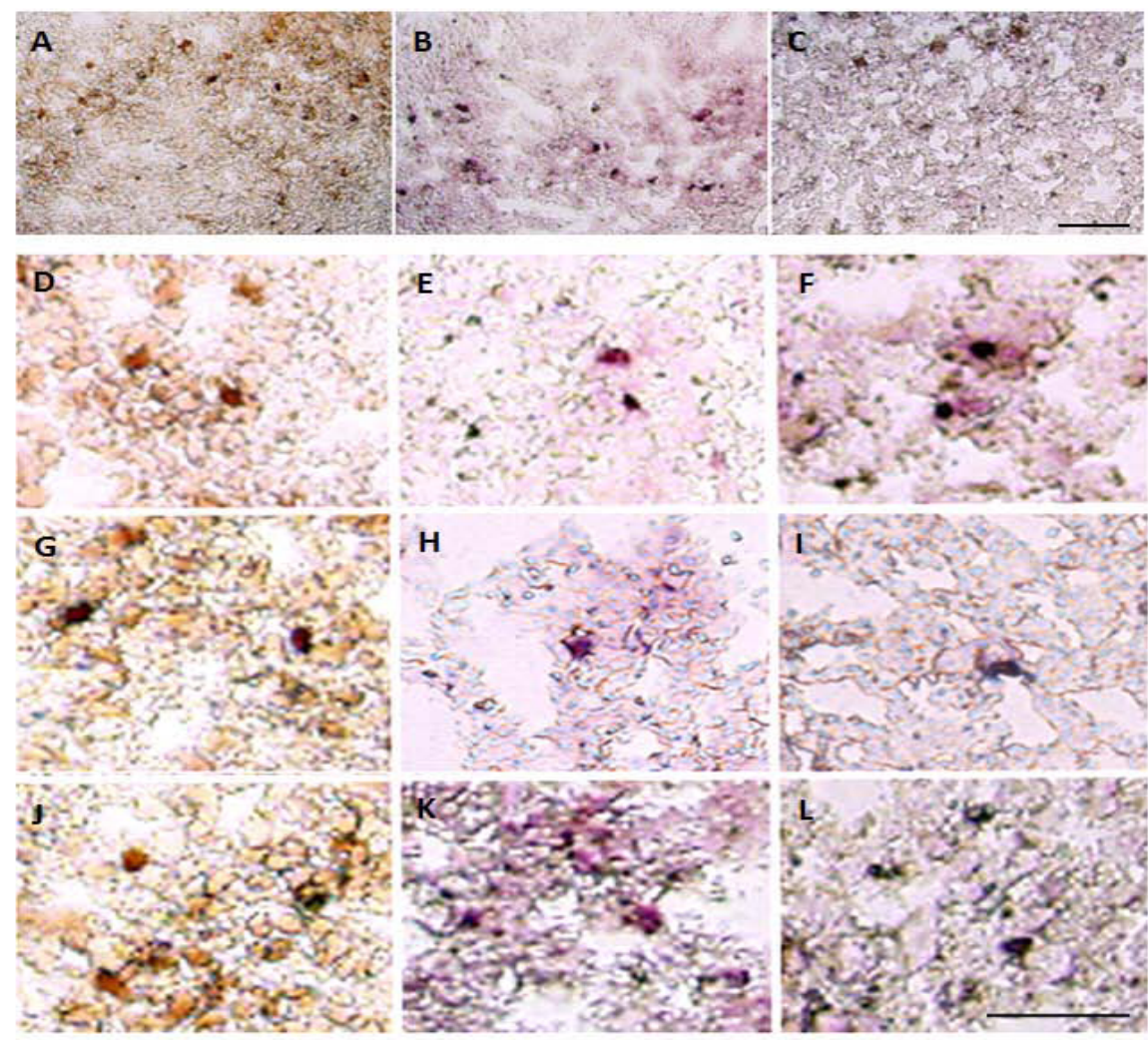

Figure 6: Immunohistochemistry of phosphorylated tau protein and calmodulin-like skin protein (CLSP). Phosphorylated tau staining (brown color; pales A, D, G and J), CLSP staining (purple color; panels $\mathrm{B}, \mathrm{E}, \mathrm{H}$ and $\mathrm{K}$ ) and the double staining (merged color; panels $\mathrm{C}, \mathrm{F}, \mathrm{I}$ and $\mathrm{L}$ ). Scale bar: $50 \mu \mathrm{m}$.

\begin{tabular}{|l|l|l|l|l|l|}
\hline Purification Step & Total protein(mg) & Total activity(units) & Specific activity(Units/mg) & Purification(fold) & Yield(\%) \\
\hline NaCl Extract & 200 & 2200 & 11 & 1 \\
\hline DEAE-FastFlow & 20 & 1066 & 53 & 5 \\
\hline ResourceQ & 3 & 1000 & 333 & 40 \\
\hline Hydroxy Apatite & 0.15 & 350 & 2333 & 30 \\
\hline ATPagarose & 0.03 & 222 & 6938 & 212 \\
\hline
\end{tabular}

One unit is defined as the amount of factor that produces a $50 \%$ reduction in the control alpha-factor precursor band under the assay conditions

Table 1: Purification of unfoldin from yeast microsome salt extract.

\begin{tabular}{|l|l|}
\hline \multicolumn{1}{|c|}{ Proteins } & Species \\
\hline Actin interacting protein2/D-lactate dehydrogenase & S. cerevisiae \\
\hline Keratin 1 & Homo sapiens \\
\hline Keratin 10 & Homo sapiens \\
\hline Small proline-rich protein & Homo sapiens \\
\hline Calmodulin-like skin protein & Homo sapiens \\
\hline TY 1B & S.cerevisiae \\
\hline
\end{tabular}

Table 2: Identified Proteins in LC-MS/MS shotgun system.

including sample pretreatment with chemical denaturing agents or detergents, are not effective for such highly aggregated structures.

Making use of the unprecedented protein-unfolding/solubilizing properties of Unfoldin, we examined the contents of laser microdissected purified PBs from a patient with Pick's disease using the LC-MS/MS shotgun method and found a novel biomarker candidate CLSP [7]. CLSP, also known as calmodulin-like 5, is a 146 amino acid protein that contains four EF-hand domains [18] and shares functional similarity with calmodulin. CLSP interacts with TGase3 [19,20], which is expressed highly in the amygdala, as shown by reverse transcriptionPCR and is also relatively strongly detected in the corpus callosum and epithelial cells [21]. The TGase family of enzymes, of which seven different members are known in the human genome, participate in many biological processes involving cross-linking proteins into large macromolecular assemblies $[19,20]$. It is expected that substantial increases in total TGase activity could occur in normal or abnormal neuronal cells because of proteolytic activation of TGases 1 and/or 3 [21]. We report that CLSP is a novel candidate Pick's disease biomarker and colocalized with phosphorylated tau protein in inclusion bodies 
known as PBs; however, for the sample limitation, in this study we could not clarify the colocalization using serial tissue sections. Even so, the double staining of the proteins (phosphorylated tau protein and CLSP) clearly merged and revealed that both proteins located on the same PBs.

Although the physiological role of CLSP in the central nervous system has not been clarified yet, our novel findings open the way for insight into the molecular mechanisms of Pick's disease.

\section{Acknowledgements}

We thank $Y$. Kozuka and $\mathrm{H}$. Sasaki for technical assistance. This study was supported by research and development projects for applications in promoting new forestry and fisheries and the Ministry of Health, Labor, and Welfare of Japan.

\section{References}

1. Hardin S, Schooley B (2002) A story of Pick's disease: a rare form of dementia. J Neurosci Nurs 34: 117-122.

2. Zhukareva V, Mann D, Pickering-Brown S, Uryu K, Shuck T, et al. (2002) Sporadic Pick's disease: a tauopathy characterized by a spectrum of pathological tau isoforms in gray and white matter. Ann Neurol 51: 730-739.

3. Delacourte A, Sergeant N, Wattez A, Gauvreau D, Robitaille Y (1998) Vulnerable neuronal subsets in Alzheimer's and Pick's disease are distinguished by their tau isoform distribution and phosphorylation. Ann Neurol 43: 193-204.

4. Arai T, Ikeda K, Akiyama H, Shikamoto Y, Tsuchiya K, et al. (2001) Distinct isoforms of tau aggregated in neurons and glial cells in brains of patients with Pick's disease, corticobasal degeneration and progressive supranuclear palsy. Acta Neuropathol 101: 167-173.

5. Hachiya NS, Ohkubo T, Kozuka Y, Yamazaki M, Mori O, et al. (2005) More than a 100 -fold increase in immunoblot signals of laser-microdissected inclusion bodies with an excessive aggregation property by oligomeric actin interacting protein 2/D-lactate dehydrogenase protein 2. Anal Biochem 347: 106-111.

6. Hachiya NS, Kaneko K (2007) Investigation of laser-microdissected inclusion bodies. Methods Cell Biol 82: 355-375.

7. Méhul B, Bernard D, Simonetti L, Bernard MA, Schmidt R (2000) Identification and cloning of a new calmodulin-like protein from human epidermis. J Bio Chem 275: 12841-12847.

8. Tsukita S, Tsukita S (1985) Desmocalmin: a calmodulin-binding high molecular weight protein isolated from desmosomes. J Cell Biol 101: 2070-2080.
9. Waters MG, Blobel G (1986) Secretory protein translocation in a yeast cell-free system can occur posttranslationally and requires ATP hydrolysis. J Cell Biol 102: 1543-1550.

10. Waters MG, Chirico WJ, Blobel G (1986) Protein translocation across the yeast microsomal membrane is stimulated by a soluble factor. J Cell Biol 103: 2629 2636.

11. Jeitner TM, Pinto JT, Krasnikov BF, Horswill M, Cooper AJ (2009) Transglutaminases and neurodegeneration. J Neurochem 109 Suppl 1: 160166.

12. Martin A, De Vivo G, Gentile V (2011) Possible role of the transglutaminases in the pathogenesis of Alzheimer's Disease and other Neurodegenerative diseases. Int J Alzheimer's Dis 2011: 865432.

13. Hachiya NS, Sakasegawa Y, Jozuka A, Tsukita S, Kaneko K (2004) Interaction of D-lactate dehydrogenase protein 2 (Dld2p) with F-actin: implication for an alternative function of Dld2p. Biochem Biophys Res Commun 319: 78-82.

14. Hachiya NS, Sakasegawa Y, Sasaki H, Jozuka A, Tsukita S, et al. (2004) Oligomeric Aip2p/DId2p forms a novel grapple-like structure and has an ATPdependent F-actin conformation modifying activity in vitro. Biochem Biophys Res Commun 320: 1271-1276.

15. Hachiya NS, Sakasegawa Y, Sasaki H, Jozuka A, Tsukita S, et al. (2004) Oligomeric Aip $2 p / D I d 2 p$ modifies the protein conformation of both properly folded and misfolded substrates in vitro. Biochem Biophys Res Commun 323 339-344.

16. Doyle T, Botstein D (1996) Movement of yeast cortical actin cytoskeleton visualized in vivo. Proc Natl Acad Sci U S A 93: 3886-3891.

17. Rozengurt N, Wu SV, Chen MC, Huang C, Sternini C, et al. (2006) Colocalization of the alpha-subunit of gustducin with PYY and GLP-1 in $L$ cells of human colon. Am J Physiol Gastrointest Liver Physiol 291: G792-G802.

18. Babini E, Bertini I, Capozzi F, Chirivino E, Luchinat C (2006) A structural and dynamic characterization of the EF-hand protein CLSP. Structure 14: 10291038.

19. Méhul B, Bernard D, Brouard M, Delattre C, Schmidt R (2006) Influence of calcium on the proteolytic degradation of the calmodulin-like skin protein (calmodulin-like protein 5) in psoriatic epidermis. Exp Dermatol 15: 469-477.

20. Méhul B, Bernard D, Schmidt R (2001) Calmodulin-like skin protein: a new marker of keratinocyte differentiation. J Invest Dermatol 116: 905-909.

21. Kim SY, Grant P, Lee JH, Pant HC, Steinert PM (1999) Differential expression of multiple transglutaminases in human brain. Increased expression and crosslinking by transglutaminases 1 and 2 in Alzheimer's disease. J Biol Chem 274 30715-30721. 